\title{
The mechanism by which P250L mutation impairs flavivirus-NS1 dimerization: an investigation based on molecular dynamics simulations
}

\author{
Edson R. A. Oliveira ${ }^{1} \cdot$ Ricardo B. de Alencastro $^{1} \cdot$ Bruno A. C. Horta $^{1}$
}

Received: 1 March 2016 / Revised: 23 May 2016 / Accepted: 2 June 2016 / Published online: 20 June 2016

(C) European Biophysical Societies' Association 2016

\begin{abstract}
The flavivirus non-structural protein 1 (NS1) is a conserved glycoprotein with as yet undefined biological function. This protein dimerizes when inside infected cells or associated to cell membranes but also forms lipidassociated hexamers when secreted to the extracellular space. A single amino acid substitution (P250L) is capable of preventing the dimerization of NS1 resulting in lower virulence and slower virus replication. In this work, based on molecular dynamics simulations of the dengue- 2 virus NS1 $\beta$-ladder monomer as a core model, we found that this mutation can induce several conformational changes that importantly affect critical monomer-monomer interactions. Based on additional simulations, we suggest a mechanism by which a highly orchestrated sequence of events propagate the local perturbations around the mutation site towards the dimer interface. The elucidation of such a mechanism could potentially support new strategies for rational production of live-attenuated vaccines and highlights a step forward in the development of novel antiflavivirus measures.
\end{abstract}

Keywords Flavivirus - Molecular dynamics simulation · NS1 $\cdot$ Virology $\cdot$ Vaccine $\cdot$ Antivirals

Electronic supplementary material The online version of this article (doi:10.1007/s00249-016-1147-9) contains supplementary material, which is available to authorized users.

Bruno A. C. Horta

bruno.horta@gmail.com

1 Instituto de Química, Universidade Federal do Rio de Janeiro, Av. Athos da Silveira Ramos, 149 - Centro de Tecnologia, Bloco A, Sala 609, Cidade Universitária, Rio de Janeiro, RJ 21941-909, Brazil
The flaviviruses comprise etiologic agents such as dengue, West Nile, and yellow fever viruses. These viruses are transmitted to humans by arthropods and are considered major health issues due to their epidemiological relevance in tropical and subtropical regions of the globe (Bhatt et al. 2013; Petersen et al. 2013; Monath and Vasconcelos 2015). During infection, the flavivirus genome (a positive sense single-stranded RNA) encodes for a polyprotein that is cleaved by host and virus proteases resulting in ten viral proteins: three structural (E, prM, and $\mathrm{C})$ and seven nonstructural (NS1, NS2A, NS2B, NS3, NS4A, NS4B, and NS5) (Lindenbach and Rice 2003). NS1 is a conserved flavivirus glycoprotein composed of 352 amino acids and presents a molecular weight of approximately $48 \mathrm{kDa}$. After expression, NS1 dimerizes and can be found in vesicular compartments within the host cell, associated to cell membranes or secreted to the extracellular space as lipid-coupled hexamers (Muller and Young 2013). Among the flavivirus proteins, NS1 is the only protein with an unknown precise biological function. Nevertheless, reports have shown the participation of NS1 in many important aspects of the viral life cycle, such as replication (Mackenzie et al. 1996; Youn et al. 2013), immune modulation (Chung et al. 2006; Costa et al. 2006; Modhiran et al. 2015) and cell metabolism (Allonso et al. 2015), which make this protein an interesting target for anti-flavivirus measures.

The recent determination of the NS1 crystal structures of dengue-serotype-2 (DENV2) and West-Nile (WNV) viruses has shown that monomers are composed of three distinct domains, namely $\beta$-roll, $\beta$-ladder and wing (Fig. 1a). A total of six disulfide bonds formed by 12 conserved cysteines were found to stabilize the net structure (Wallis et al. 2004; Akey et al. 2014). A head-to-head orientation between two $\beta$-ladder domains, so as to form an extended central $\beta$-sheet region, basically defines the 

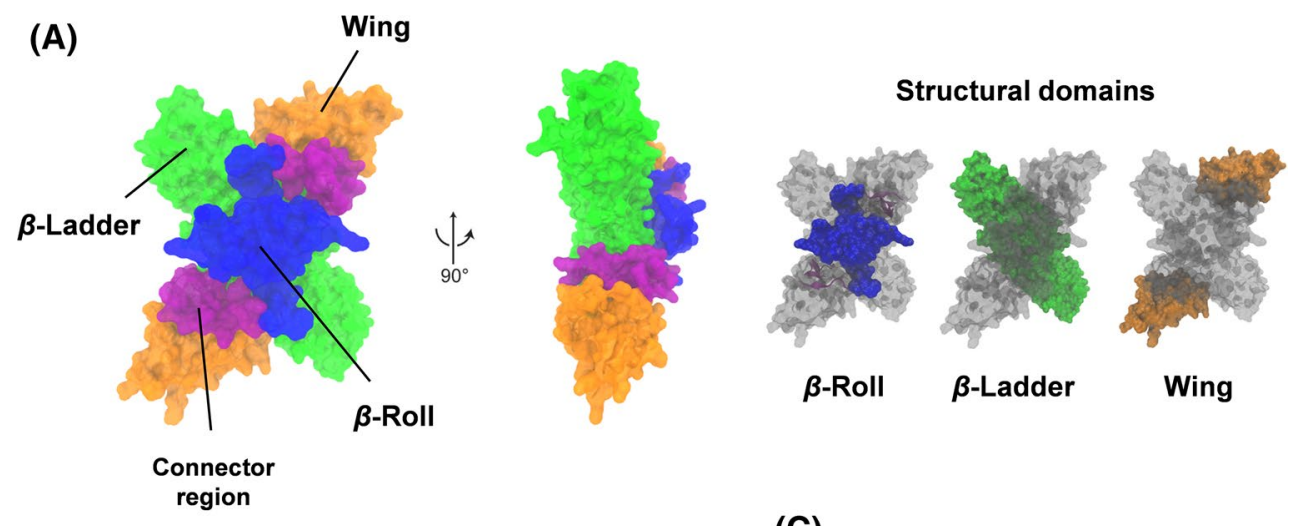

(B)
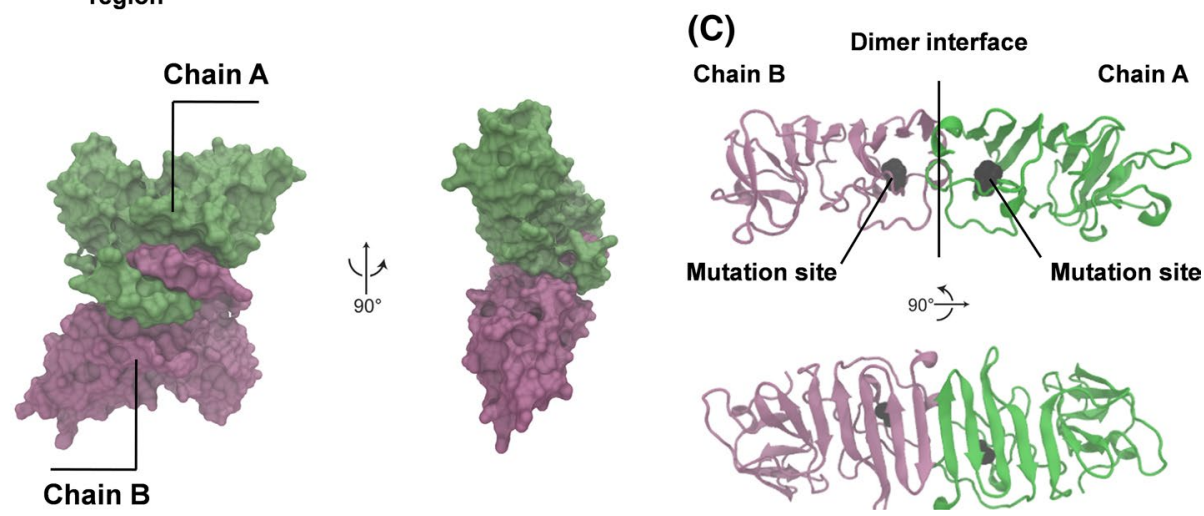

Fig. 1 Three-dimensional illustration of the NS1 dimer and its domains. The coordinates were extracted from the DENV2 NS1 crystal structure (Akey et al. 2014) under PDB code 4O6B. a On the left side, a surface representation (in two different orientations) showing the extended $\beta$-ladder in green, the $\beta$-roll in blue, the wing in orange, and the connector region in violet. On the right side, the three structural domains are shown individually (highlighted) while keeping the rest of the protein in transparent gray representation. b The surface is colored by chain (chain A in dark green and chain B in mauve), revealing the interface between the two monomers. c A cartoon representation of the $\beta$-ladder domain showing the extended $\beta$ sheet formed by the head-to-head orientation between the two monomers. The mutation site is also depicted in a black surface representation dimeric shape of NS1 (Fig. 1b). Each monomer of the $\beta$-ladder domain is composed of $9 \beta$ strands on one face and residues that lack a well-defined secondary structure on the other face. The latter is commonly referred to as the "spaghetti loop" region (Akey et al. 2014). Oligomerization of NS1 is observed in all flaviviruses and the dimeric form of this protein appears to play a role in flavivirus virulence. Studies with Kunjin and Murray Valley encephalitis viruses have demonstrated that a single amino acid substitution at residue 250 from proline to leucine (P250L) is critical for loss of NS1 dimerization (Hall et al. 1999; Clark et al. 2007). Additionally, according to these reports, the P250L mutation is correlated with retarded virus growth and reduced virulence in mice.

Structurally, residue P250 is located on the "spaghetti loop" region of the $\beta$-ladder domain, which lies beneath the $\beta$-sheet structure (Fig. 1c). This residue is not part of the dimer interface. It is thus quite intriguing that this mutation alone is sufficient to prevent dimerization. We hypothesize that this mutation triggers a chain of events leading to conformational changes in the dimer interface. Due to the high degree of sequence similarity between the NS1 proteins among the flaviviruses (Mackow et al. 1987; Deubel et al. 1988, 1989; Wright and Cauchi 1989), it is likely that the experimental observations found for Kunjin and Murray Valley encephalitis viruses hold also for other members. Taking this into account, we concentrated our efforts on investigating the impact of the P250L mutation on the structure of DENV2-NS1 protein using molecular dynamics (MD) simulations.

We have carried out 100-ns MD simulations considering systems composed of either the native (WT) or the mutated NS1 $\beta$-ladder monomer. Figure 2a shows the root-meansquare deviations (RMSD) calculated considering the backbone atoms of the WT and P250L monomers with respect to the crystal structure (Akey et al. 2014). The WT simulation converged to a RMSD value of $\sim 0.11 \mathrm{~nm}$ after $\sim 0.3 \mathrm{~ns}$ and remained stable throughout the entire simulation, while the P250L simulation exhibited an accentuated increase of its RMSD value after $\sim 5 \mathrm{~ns}$ stabilizing at a deviation of $\sim 0.15 \mathrm{~nm}$. These data indicate important structural changes induced by the mutation. In order to visualize the regions 

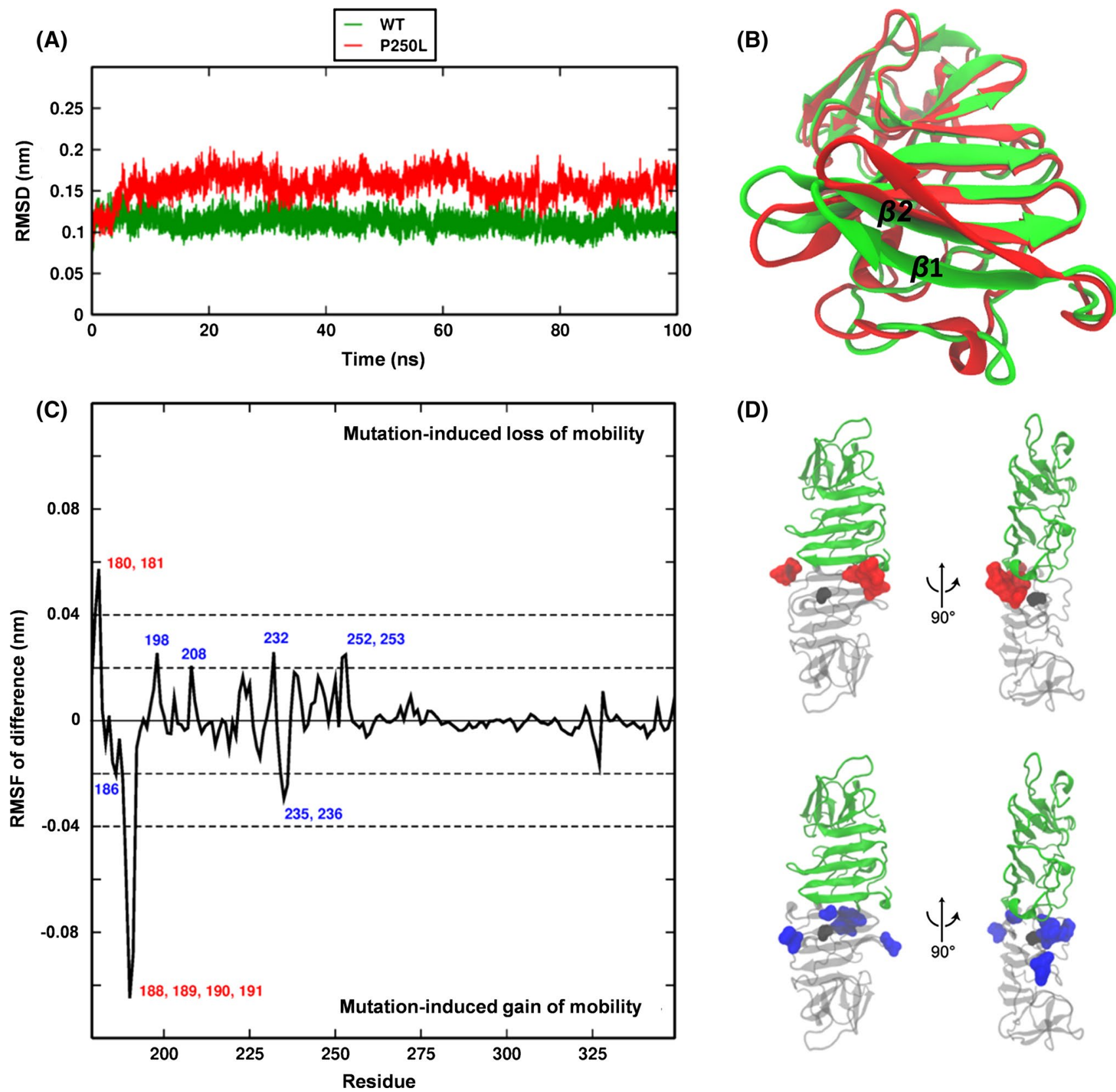

(D)

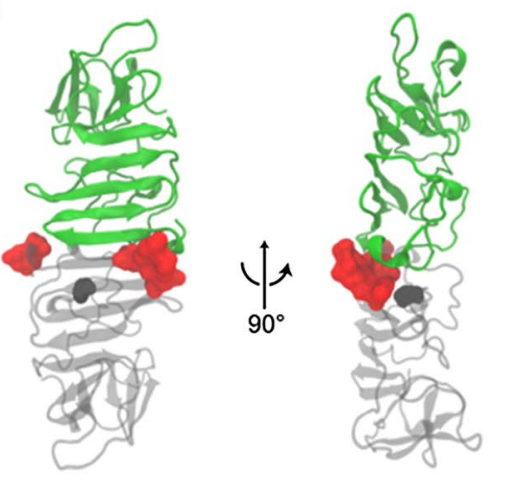

Fig. 2 General structural features of WT and P250L $\beta$-ladder domains of NS1 on the course of the 100-ns molecular dynamics simulations. a The backbone root-mean-square deviations (RMSD) with respect to the crystal structure (Akey et al. 2014). b The superposed final structures (WT in green, P250L in red) after least-squares fitting based on all backbone atoms. $\mathbf{c}, \mathbf{d}$ The difference in root-mean-square

fluctuations (RMSF) between WT and P250L averaged over the last $50 \mathrm{~ns}$ of the simulations, along with the cartoon representation of the extended $\beta$-sheet region highlighting the most affected residues. Residues with major changes in RMSF are shown in blue (to a lesser extent) and in red (to a higher extent). The mutation site (P250L substitution) is represented in black

of the protein where these major structural changes took place, we superposed the final structures of the two simulations using least-squares fitting based on all backbone atoms (Fig. 2b). A visual inspection of the final structure clearly suggested that the most affected regions are the ones responsible for the monomer-monomer contact upon dimerization (interface region) with almost no disturbance of the regions away from the interface. Note that the effect of the mutation is more pronounced on the a priori more rigid $\beta$-sheet structure, in particular the $\beta 1$ strand, compared to the more flexible loop region comprising the so called "spaghetti loop" located beneath the $\beta$-sheet. The root-mean-square fluctuations (RMSF) were calculated on a per-residue basis for each system considering the last 50 
(A)

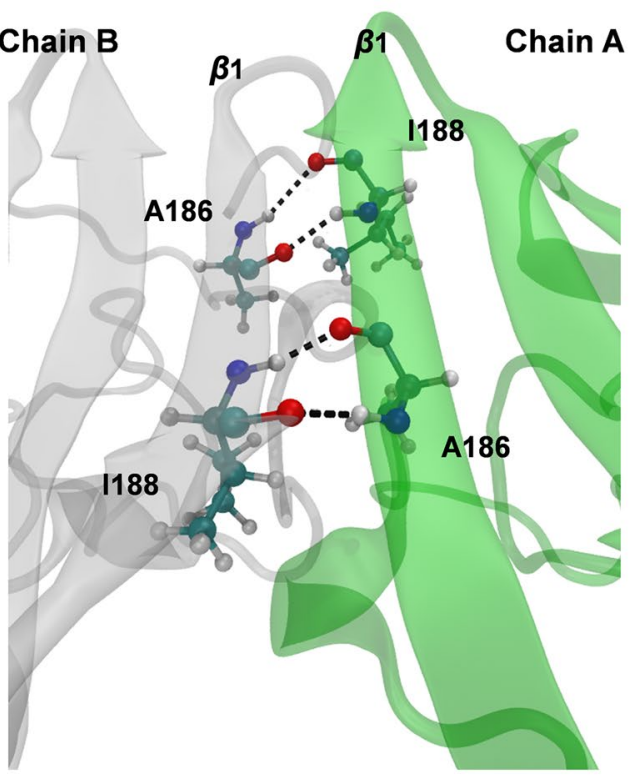

(B)

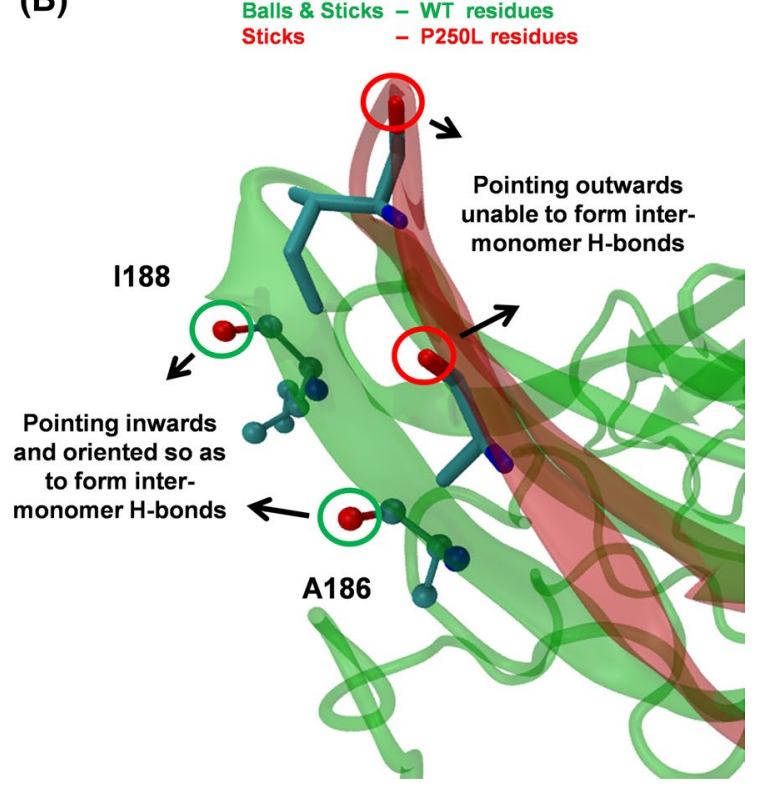

are represented by dashed lines. b Least-squares-fitting superposition based on all backbone atoms of the final structures (WT and P250L) after the 100-ns molecular dynamics simulations. A large conformational change involving the $\beta 1$-strand of the mutant with respect to the WT is apparent, resulting in major orientational changes of the carbonyl groups of residues A186 and I188 ns of the simulation trajectories and based on the backbone atoms after least-squares fitting to the average structures. The relative differences between the RMSF of the two systems $\left(\mathrm{RMSF}_{\mathrm{WT}}-\mathrm{RMSF}_{\mathrm{P} 250 \mathrm{~L}}\right)$ are displayed in Fig. 2c and $\mathrm{d}$, where positive and negative values indicate loss and gain of mobility, respectively, upon mutation. At this point, we gathered a number of places of evidence that the single point mutation P250L can lead to significant conformational changes in the interface region.

Our next step consisted of investigating how these conformational alterations might structurally impair the NS1 dimer formation. A visual inspection of the WT $\beta$-ladder crystal structure revealed the amino acids that interact with their counterpart chain to provide a stable dimer. Residues A186 and I188, present in the $\beta 1$-strand, expose donor and acceptor atoms towards the interface in order to form hydrogen bonds that could critically contribute to the headto-head intermonomer interactions (Fig. 3a). As a consequence of the $\beta 1$-strand conformational change, residues A186 and I188 have lost their optimal orientation to establish inter-chain hydrogen bonds (Fig. 3b). Regardless of the importance of additional intermolecular interactions that stabilize the NS1 dimer, such as other hydrogen bonds and hydrophobic interactions located in the $\beta$-roll domain, the head-to-head engagement between the $\beta$-ladder monomers may represent the major component contributing to dimer stability. The expression of WNV or DENV-1 NS1 $\beta$-ladder monomers (C-terminal ${ }_{172-352}$ region) alone are enough to produce stable head-to-head dimers, as detected in the crystal lattice and in solution (Edeling et al. 2014). Therefore, in agreement with previous experimental reports (Hall et al. 1999; Clark et al. 2007), the P250L mutation-induced distortions of the $\beta 1$-strand within the mutated monomer would in fact be a possible explanation for the inhibition of NS1 dimer formation.

After showing the consequences of P250L mutation on the structure of NS1, mainly displacing the $\beta 1$-strand and impairing important monomer-monomer contacts, the question that arises is how a single mutation of a residue that is not at the interface is producing such a dramatic effect. After analyzing the simulation trajectories in terms of the distances between the mutation site and residues A186 and I188 (Fig. S1), we observed that this process occurred in a relatively short time scale (within the first 7-8 ns). By visually inspecting the trajectory frames and by monitoring several distances between residues around the mutation site (Fig. S2), we were able to identify the events preceding the complete distortion. Very early $(\sim 27 \mathrm{ps})$ in the trajectory of the mutated NS1, we found that the presence of L250 induced a conformational change of H195, significantly displacing it from its original position, a trigger that has been already speculated previously (Edeling et al. 2014). The side chain of the mutated residue, probably by means of a steric effect, pushed away the imidazole ring of H195 


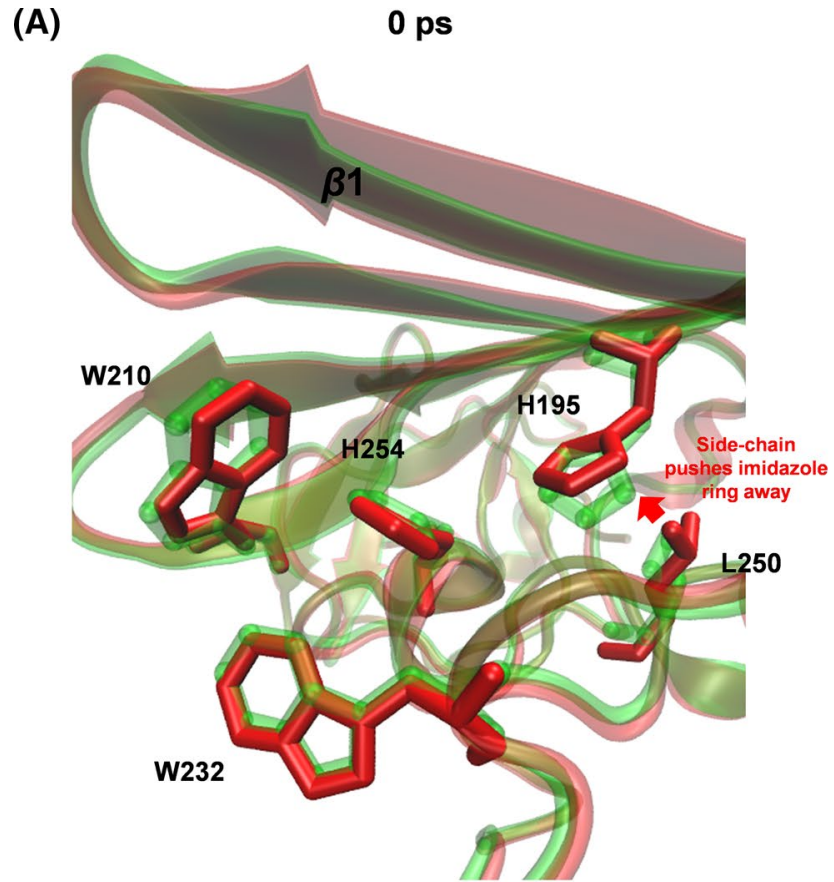

Fig. 4 A representation of the early stages of the proposed mechanism for the $\beta 1$-strand displacement induced by the P250L mutation. The P250L (red) and WT (green; for comparison purposes) NS1 $\beta$-ladder monomers are shown as superposed structures after leastsquares fitting based on all backbone atoms. a The initial structures (after thermalization) suggest the pushing effect of the more bulky L250 residue on the side chain of H195. b The structures after 360 ps of molecular dynamics simulations. The numbers within circles represent the time evolution of the conformational changes. The first

moving it towards the $\mathrm{H} 254$ residue. This fact, in turn, may have led to a transient conformational instability of the side chain of the neighboring $\mathrm{H} 254$, promoting a displacement of residue W232 away from the interface $(\sim 360 \mathrm{ps})$ and making room for W210 to partially occupy the space previously filled by W232 (Fig. 4). These early stages of the mechanism produced a state in which W232 became fully exposed to the solvent and W210 tended to enter deeper inside the cavity left by W232. From this moment on, W210 assumed a conformation in which its large side chain was almost orthogonal to the W232 side chain and tended to push the $\beta 1$-strand upwards. This lasted for 7-8 ns and was followed by the previously described distortion of the $\beta 1$ strand. Therefore, we hypothesize that this upward pressure exerted by W210 on the $\beta 1$-strand, which is only possible after the displacement of W232, is the main effect that promoted this distortion.

Based on the above observations, we carried out several additional 50- ns MD simulations in order to verify the role of residues H195, H254, W210, and W232. Below we enumerate several hypotheses followed by our observations and conclusions from the analysis of these simulations:
(B) 360 ps

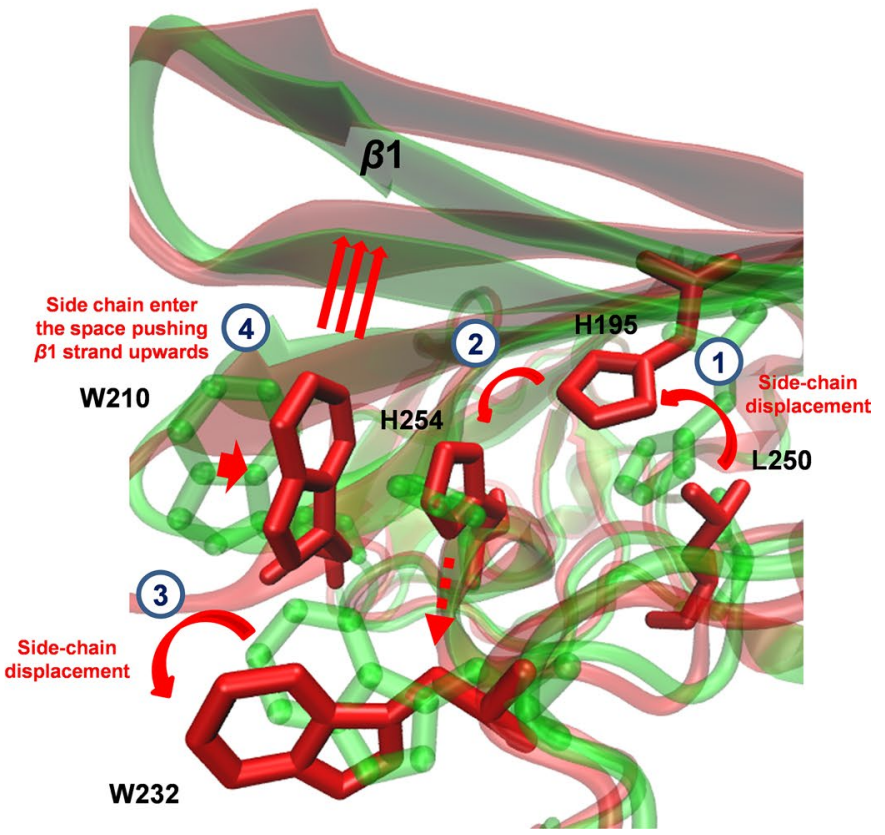

event starts just after thermalization and is completed around $27 \mathrm{ps.} \mathrm{It}$ corresponds to the side-chain displacement of H195 caused by L250. As a consequence, the side chain of $\mathrm{H} 254$ undergoes a conformational change, probably due to a steric effect (step 2). The perturbation on residue $\mathrm{H} 254$ probably induces the displacement of W232, exposing it to the solvent (step 3). As a final step, W210 partially occupies the hydrophobic pocket previously filled by W232, pushing the $\beta 1$-strand upwards

1. If the altered conformation of the imidazole ring of H195 upon P250L mutation somehow disturbed the conformation of $\mathrm{H} 254$, a mutation of $\mathrm{H} 195$ by a small and less interacting residue such as Ala, would suppress the whole process of conformational change. In order to check this hypothesis, we simulated the double mutant P250L-H195A. As expected, this double mutant did not evidence any sign of $\beta 1$-strand distortion, indicating that H195A suppresses the effect of P250L (Fig. S3, panels A and B).

2. If the instability of $\mathrm{H} 254$ was somehow related to the conformational change that exposed residue W232 to the solvent, a mutation of $\mathrm{H} 254$ to Ala would suppress the effect of P250L. Therefore, we simulated the double mutant P250L-H254A. Analogously to the case above, this double mutant also did not evidence any sign of $\beta 1$-strand distortion, indicating that $\mathrm{H} 254 \mathrm{~A}$ also suppresses the effect of P250L (Fig. S3, panels A and C).

3. If the role of $\mathrm{W} 232$ upon the $\mathrm{P} 250 \mathrm{~L}$ mutation was to move away from the interface towards the solvent, liberating space so that W210 is able to enter and push the 
$\beta 1$ strand upwards, then a mutation of W232 to a small residue such as Ala would be enough to promote the same effect as P250L. In order to check this hypothesis, we constructed the mutant W232A without mutating P250. The effect was exactly what we expected. With Ala at position 232, W210 rapidly entered the space and assumed the same position as in the simulation of P250L (Fig. S3, panels D and E). After $\sim 11$ $\mathrm{ns}$, it promoted the distortion of the $\beta 1$ strand. This result strongly supports our mechanistic proposal and suggests a second mutation that could independently impair NS1 dimerization.

4. Finally, if the mutation P250L culminated with W210 occupying the space of W232 and pushed the $\beta 1$-strand upwards, then the double mutant P250L-W210A would not result in the $\beta 1$-strand distortion. The simulation of this double mutant was also carried out, showing that W210A mutation also suppresses the effect of P250L (Fig. S3, panels D and F).

The primary sequences of the $\beta$-ladder domains of different flaviviruses presenting epidemiological interest were aligned to verify the conservation level of these particular residues (H195, H254, W232, and W210). In line with our results, the amino acids that apparently orchestrate the mechanism that disrupts the conformation of the $\beta 1$-strand as a result of the $\mathrm{P} 250 \mathrm{~L}$ substitution were found to be highly conserved (Fig. S4). This fact supports the robustness of the proposed mechanism and also suggests that it may occur among other flaviviruses.

An interesting feature of the flavivirus NS1 arises from its physico-chemical nature. Because of hydrophilic and hydrophobic regions over the NS1 surface, an assumed peculiarity of this protein when interacting with biological membranes is its orientation. It is postulated that the $\beta$ -roll domain would function as a "hydrophobic protrusion" to attach NS1 to lipid membranes (Akey et al. 2014). As a direct consequence, this would orientate the other face (the "spaghetti loop" region) towards the extracellular space, exposing this area for immune recognition (Akey et al. 2015). We suggest that the inhibition of NS1 dimerization would affect its membrane-binding capacity due to the disruption of the "hydrophobic protrusion" composed by monomer-complementary regions of the $\beta$-roll domains.

Anti-flavivirus measures have become increasingly needed since classical flavivirus diseases, e.g., dengue and West Nile fevers, are still in need of specific measures for their mass prevention (Ishikawa et al. 2014) amongst humans. Apart from this, another emerging disease caused by a member of the flaviviruses, Zika virus, is a growing threat in tropical and sub-tropical countries (Cardoso et al. 2015; Campos et al. 2015), reinforcing even more the necessity of developing such countermeasures. Historically, anti-measles, anti-mumps, and anti-rubella are successful examples of live-attenuated vaccines, however, an important drawback regarding their production is the lack of a rational strategy for attenuation (Minor 2015; Vignuzzi and Wendt 2008). The mechanism for the effects of the P250L substitution proposed here suggests how a single amino acid change in a specific flavivirus protein may act preventing its dimerization. Knowing that NS1-dimer integrity is important for flavivirus virulence, we envision chemically driven or site-directed mutagenesis as interesting tools for vaccine development. Besides the $\mathrm{P} 250 \mathrm{~L}$ mutation, we found W232A to be another promising alternative to prevent NS1 dimerization considering that the simulation results show similar conformational changes in the dimer interface. Given the risk of attenuated viruses to revert to the wild-type pathogenic form (Minor 2015), the presence of both mutations could be highly advantageous to provide an extra layer of vaccine safety to guard against reversion. Such knowledge of potential mutations that result in virus debilitation would involve a less empirical and a more rational practice in the development of a live-attenuated vaccine.

\section{Computational details}

\section{Simulated systems}

The systems considered in the present study are the wildtype and mutated monomers of the NS1 $\beta$-ladder $179-349$ domain of the DENV2 serotype. The initial coordinates corresponding to the wild-type structure were extracted from the chain A of the previously determined crystal structure deposited under PDB code 4O6B (Akey et al. 2014). Only residues 179 to 349 composed the core model and the glycosylation at position 207 was not considered. The missing atoms were added using the Swiss PDB Viewer (SPDBV) program (Guex and Peitsch 1997). This structure served as basis for the construction of the mutant models P250L，P250L-H195A，P250L-H254A，P250L-W210A, and W232A. The mutations were performed using the SPDBV program.

\section{Simulation protocol}

The MD simulations were performed using the Gromacs simulation package (Berendsen et al. 1995; Hess et al. 2008) together with the AMBER ff99SB-ILDN force field (Lindorff-Larsen et al. 2010) and the TIP3P water model (Jorgensen et al. 1983). The hydrogen atoms were added according to a neutral $\mathrm{pH}$ condition. The simulations were carried out under periodic boundary conditions based on rectangular boxes. Newton's equations of motion were 
Table 1 Simulated systems and simulation conditions

\begin{tabular}{llllll}
\hline System & $N_{\text {prot }}$ & $M_{\text {wat }}$ & $N_{\mathrm{Na}^{+}}$ & $N_{\text {total }}$ & Duration (ns) \\
\hline WT & 2664 & 11,018 & 1 & 35,714 & 100 \\
P250L & 2669 & 11,017 & 1 & 35,721 & 100 \\
P250L-H195A & 2662 & 10,950 & 1 & 35,513 & 50 \\
P250L-H254A & 2662 & 10,945 & 1 & 35,498 & 50 \\
W232A & 2650 & 10,953 & 1 & 35,510 & 50 \\
P250L-W210A & 2655 & 10,948 & 1 & 35,500 & 50 \\
\hline
\end{tabular}

The different columns report the simulated system (system), the number of protein atoms $\left(N_{\text {prot }}\right)$, the number of water molecules $\left(M_{\text {wat }}\right)$, the number of $\mathrm{Na}^{+}$ions $\left(N_{\mathrm{Na}^{+}}\right)$, the total number of atoms $\left(N_{\text {total }}\right)$ and the simulation length (duration)

integrated using the leapfrog scheme (Hockney 1970) with a time step of 2 fs. All bonds involving hydrogen atoms were constrained using the LINCS procedure (Hess et al. 1997). A cutoff distance of $10 \AA$ was used for the truncation of the Lennard-Jones interaction and the shortrange electrostatic interactions. The smooth particle mesh Ewald (SPME) method (Essmann et al. 1995) was used with a $32 \times 32 \times 32$ grid and a fourth-order interpolation scheme to compute the long-range electrostatic interactions. The pairlists were generated and updated according to the Verlet scheme (Páll and Hess 2013). The center of mass motion was removed every 100 ps. The systems were energy minimized with 5000 steps of the Steepestdescent algorithm and subsequently equilibrated. The equilibration was performed using positional restraints on the protein atoms and lasted $300 \mathrm{ps}$. The simulations were performed in the NPT ensemble (constant number of particles $N$, pressure $P$, and temperature $T$ ) with a reference pressure $P$ of 1 bar and reference temperature $T$ of $310 \mathrm{~K}$. The temperature was maintained by a velocity-rescaling algorithm (Bussi et al. 2007) with the protein and solvent degrees of freedom separately coupled to a temperature bath at temperature $T$, with a coupling constant of $0.1 \mathrm{ps}$. Aside from the equilibration that was performed using a Berendsen thermostat (Berendsen et al. 1984) with compressibility of $4.510^{-5} \mathrm{bar}^{-1}$ and relaxation time of $2 \mathrm{ps}$, the pressure was maintained close to the reference pressure $P$ by isotropic coupling using the Parrinello-Rahman algorithm (Parrinello and Rahman 1980). The production runs had a duration of $100 \mathrm{~ns}$ for systems WT and P250L and $50 \mathrm{~ns}$ for systems P250L-H195A, P250L-H254A, P250L-W210A, and W232A (Table 1). The equilibration steps were not considered for analysis and are not represented in the time series.

Acknowledgments This work was supported by the Brazilian foundations CAPES, CNPq and by the Rio de Janeiro state agency FAPERJ. E. R. A. O. also acknowledges CAPES for his post-doctoral fellowship.

\section{References}

Akey DL, Brown WC, Dutta S, Konwerski J, Jose J, DelProposto Jurkiw TJ, Ogata J, Skiniotis CM, Kuhn G, Smith RJ (2014) Flavivirus NS1 structures reveal surfaces for associations with membranes and the immune system. Science 343:881-885

Akey DL, Brown WC, Jose J, Kuhn RJ, Smith JL (2015) Structureguided insights on the role of NS1 in flavivirus infection. Bioessays 37:489-494

Allonso D, Andrade IS, Conde JN, Coelho DR, Rocha DC, da Silva ML, Ventura GT, Silva EM, Mohana-Borges R (2015) Dengue virus NS1 protein modulates cellular energy metabolism by increasing glyceraldehyde-3-phosphate dehydrogenase activity. J Virol 89:11871-11883

Berendsen HJC, Postma JPM, van Gunsteren WF, di Nola A, Haak JR (1984) Molecular dynamics with coupling to an external bath. J Chem Phys 81:3684-3690

Berendsen HJC, van der Spoel D, van Drunen R (1995) GROMACS: a message-passing parallel molecular dynamics implementation. Comput Phys Commun 91:43-56

Bhatt S, Gething PW, Brady OJ, Messina JP, Farlow AW, Moyes CL, Drake JM, Brownstein JS, Hoen AG, Sankoh O, Myers MF, George DB, Jaenisch T, Wint GR, Simmons CP, Scott TW, Farrar JJ, Hay SI (2013) The global distribution and burden of dengue. Nature 496:504-507

Bussi G, Donadio D, Parrinello M (2007) Canonical sampling through velocity rescaling. J Chem Phys, 126, 014101/1-014101/7

Campos GS, Bandeira AC, Sardi SI (2015) Zika virus outbreak, Bahia, Brazil. Emerg Infect Dis 21:1885-1886

Cardoso CW, Paploski IAD, Kikuti M, Rodrigues MS, Silva MMO, Campos GS, Sardi SI, Kitron U, Reis MG, Ribeiro GS (2015) Outbreak of exanthematous illness associated with Zika, Chikungunya, and Dengue viruses, Salvador, Brazil. Emerg Infect Dis 21:2274-2276

Chung KM, Liszewski MK, Nybakken G, Davis AE, Townsend RR, Fremont DH, Atkinson JP, Diamond MS (2006) West Nile virus nonstructural protein NS1 inhibits complement activation by binding the regulatory protein factor $\mathrm{H}$. Proc Natl Acad Sci USA 103:19111-19116

Clark DC, Lobigs M, Lee E, Howard MJ, Clark K, Blitvich BJ, Hall RA (2007) In situ reactions of monoclonal antibodies with a viable mutant of Murray Valley encephalitis virus reveal an absence of dimeric NS1 protein. J Gen Virol 88:1175-1183

Costa SM, Freire MS, Alves AM (2006) DNA vaccine against the non-structural 1 protein (NS1) of dengue 2 virus. Vaccine 24:4562-4564

Deubel CW, Heinz FX, Stöckl E, Kunz C (1989) Genome sequence of tick-borne encephalitis virus (Western subtype) and comparative analysis of nonstructural proteins with other flaviviruses. Virology 173:291-301

Deubel V, Kinney RM, Trent DW (1988) Nucleotide sequence and deduced amino acid sequence of the nonstructural proteins of dengue type 2 virus, Jamaica genotype: comparative analysis of the full-length genome. Virology 165:234-244

Edeling MA, Diamond MS, Fremont DH (2014) Structural basis of Flavivirus NS1 assembly and antibody recognition. Proc Natl Acad Sci USA 111:4285-4290

Essmann U, Perera L, Berkowitz ML, Darden T, Lee H, Pedersen LG (1995) A smooth particle mesh Ewald method. J Chem Phys 103:8577-8593

Guex N, Peitsch MC (1997) Swiss-Model and the Swiss-PdbViewer: an environment for comparative protein modeling. Electrophoresis 18:2714-2723

Hall RA, Khromykh AA, Mackenzie JM, Scherret JH, Khromykh TI, Mackenzie JS (1999) Loss of dimerisation of the nonstructural 
protein NS1 of Kunjin virus delays viral replication and reduces virulence in mice, but still allows secretion of NS1. Virology 264:66-75

Hess B, Bekker H, Berendsen HJC, Fraaije JGEM (1997) LINCS: a linear constraint solver for molecular simulations. J Comput Chem 18:1463-1472

Hess B, Kutzner C, van der Spoel D, Lindahl E (2008) GROMACS 4: algorithms for highly efficient, load-balanced, and scalable molecular simulation. J Chem Theory Comput 4:435-447

Hockney RW (1970) The potential calculation and some applications. Methods Comput Phys 9:135-211

Ishikawa T, Yamanaka A, Konishi E (2014) A review of successful flavivirus vaccines and the problems with those flaviviruses for which vaccines are not yet available. Vaccine 32:1326-1337

Jorgensen WL, Chandrasekhar J, Madura JD, Impey RW, Klein ML (1983) Comparison of simple potential functions for simulating liquid water. J Chem Phys 79:926-935

Lindenbach BD, Rice CM (2003) Molecular biology of flaviviruses. Adv Virus Res 59:23-61

Lindorff-Larsen K, Piana S, Palmo K, Maragakis P, Klepeis JL, Dror RO, Shaw DE (2010) Improved side-chain torsion potentials for the Amber ff99SB protein force field. Proteins 78:1950-1958

Mackenzie JM, Jones MK, Young PR (1996) Immunolocalization of the dengue virus nonstructural glycoprotein NS1 suggests a role in viral RNA replication. Virology 220:232-240

Mackow E, Makino Y, Zhao BT, Zhang YM, Markoff L, BucklerWhite A, Guiler M, Chanock R, Lai CJ (1987) The nucleotide sequence of dengue type 4 virus: analysis of genes coding for nonstructural proteins. Virology 159:217-228

Minor PD (2015) Live attenuated vaccines: historical successes and current challenges. Virology 479-480:379-392
Modhiran N, Watterson D, Muller DA, Panetta AK, Sester DP, Liu L, Hume DA, Stacey KJ, Young PR (2015) Dengue virus NS1 protein activates cells via Toll-like receptor 4 and disrupts endothelial cell monolayer integrity. Sci Transl Med 7, 304/1-304/10

Monath TP, Vasconcelos PF (2015) Yellow fever. J Clin Virol 64:160-173

Muller DA, Young PR (2013) The flavivirus NS1 protein: molecular and structural biology, immunology, role in pathogenesis and application as a diagnostic biomarker. Antivir Res 98:192-208

Parrinello M, Rahman A (1980) Crystal structure and pair potentials: a molecular-dynamics study. Phys Rev Lett 45:1196-1199

Petersen LR, Brault AC, Nasci RS (2013) West Nile virus: review of the literature. J Am Med Assoc 310:308-315

Páll S, Hess B (2013) A flexible algorithm for calculating pair interactions on SIMD architecture. Comput Phys Commun 184:2641-2650

Vignuzzi M, Wendt E (2008) Engineering attenuated virus vaccines by controlling replication fidelity. Nat Med 14:154-161

Wallis TP, Huang C-Y, Nimkar SB, Young PR, Gorman JJ (2004) Determination of the disulfide bond arrangement of dengue virus NS1 protein. J Biol Chem 279:20729-20741

Wright PJ, Cauchi MR, Ng ML (1989) Definition of the carboxy termini of the three glycoproteins specified by dengue virus type 2 . Virology 171:61-67

Youn S, Ambrose RL, Mackenzie JM, Diamond MS (2013) Nonstructural protein-1 is required for West Nile virus replication complex formation and viral RNA synthesis. Virol J 18, $339 / 1-339 / 14$ 